\title{
Use of Renin-Angiotensin System Blockers Increases Serum Potassium in Anuric Hemodialysis Patients
}

\author{
Ezio Movilli Corrado Camerini Paola Gaggia Roberto Zubani \\ Giovanni Cancarini \\ U.O. of Nephrology, ASST Spedali Civili and University of Brescia, Brescia, Italy
}

\section{Keywords}

Hyperkalemia · Renin angiotensin system · Hypertension ·

Dialysis · Cardiovascular

\begin{abstract}
Background: Angiotensin converting enzyme inhibitors (ACEi) and angiotensin receptor blockers (ARB) are increasingly used in uremic patients (pts). However, their effect on serum potassium (sK) concentrations in anuric pts on chronic hemodialysis treatment (HD) is controversial. The aim of the study was to evaluate sK before and after the start of ACEi/ARB therapy. Methods: In the period 1/1/2015 $31 / 12 / 2015,112$ out of 240 prevalent HD pts on thrice weekly HD treatment followed at our institution started the ACEi/ ARB therapy. The mean age was $67 \pm 14$ years, $67 / 112$ were men, dialysis vintage was 6-212 months. In the 3 months before (PRE; $\mathrm{N}^{\circ} 36 \mathrm{HD}$ sessions) and after (POST; $\mathrm{N}^{\circ} 36 \mathrm{HD}$ sessions) the start of ACEi/ARB therapy, the following variables were evaluated in pre dialysis after the long interdialysis interval: sK (mean of 12 determinations; $\mathrm{mmol} / \mathrm{L}$ ), maximum sK (maximum $\mathrm{K}$ value observed during observations;
\end{abstract}

sKmax; $\mathrm{mmol} / \mathrm{L}$ ), serum sodium ( $\mathrm{sNa}$; $\mathrm{mmol} / \mathrm{L}$ ), pre dialysis systolic blood pressure (SBP; $\mathrm{mm} \mathrm{Hg}$ ) and diastolic blood pressure (DBP; $\mathrm{mm} \mathrm{Hg}$ ), body weight (BW; $\mathrm{Kg}$ ), interdialytic weight gain (IWG; Kg), Kt/V, serum bicarbonate concentrations (sBic; $\mathrm{mmol} / \mathrm{L})$, protein catabolic rate $(\mathrm{PCRn} ; \mathrm{g} / \mathrm{KgBW} /$ day). SBP, DBP, IWG are the mean of the $24 \mathrm{HD}$ sessions. Out of 112 patients, 102 were on antihypertensive therapy. The duration of $\mathrm{HD}$ and blood and dialysate flow rates were kept constant. Data are expressed as mean \pm SD. Student $t$ test for paired and unpaired data for normally distributed variables, Mann-Whitney test for medians, $X^{2}$ test for categorical data were employed to compare groups. A significant difference was defined as $p<0.05$. Results: sK increased from $5.0 \pm$ $0.4 \mathrm{mmol} / \mathrm{L}$ PRE to $5.7 \pm 0.5 \mathrm{mmol} / \mathrm{L}$ POST $(p<0.0001)$. $\mathrm{sKmax}$ increased from $5.3 \pm 0.5 \mathrm{mmol} / \mathrm{L}$ PRE to $6.2 \pm 0.6 \mathrm{mmol} / \mathrm{L}$ POST $(p<0.0001)$. The percentage of pts with normal sK concentrations decreased from $82 \%$ PRE to $29 \%$ POST ( $p<$ 0.0001 ). Mild hyperkalemia increased from 18 to $52 \%$ ( $p<$ 0.001 ); in $31 \%$ of the patients, it was necessary to reduce the $\mathrm{K}$ dialysate concentration. None of the patients had severe hyperkalemia PRE, but 19\% developed severe hyperkalemia POST $(p<0.0001)$ necessitating treatment withdrawal. Mean

\section{KARGER}

(c) 2018 S. Karger AG, Basel

E-Mail karger@karger.com

www.karger.com/ajn
Ezio Movilli, MD

U.O. of Nephrology Spedali Civili Brescia

Piazz.le Spedali Civili 1

IT-25123 Brescia (Italy)

E-Mail eziomov@libero.it 
sK in these pts varied from $5.2 \pm 0.3 \mathrm{mmol} / \mathrm{L}$ PRE to $6.5 \pm$ $0.2 \mathrm{mmol} / \mathrm{L}$ at the moment of withdrawal $(p<0.0001)$ and sKmax from $5.5 \pm \mathrm{mmol} / \mathrm{L}$ PRE to $6.9 \pm 0.3 \mathrm{mmol} / \mathrm{L}(p<0.0001)$. After withdrawal of ACEi/ARB, sK and sKmax concentrations decreased to basal levels within 1 month. There were no significant changes of BW, IWG, SBP, DBP, Na, $\mathrm{Hb}, \mathrm{Kt} / \mathrm{V}$, sBic, and $P C R n$ in both periods. Conclusions: ACEi/ARB therapy is associated with an increased risk of hyperkalemia in anuric hemodialysis patients. The proportion of patients with normal sK concentrations decreased from 82 to $29 \%$ and with mild hyperkalemia increased from 18 to $52 \%$. Severe hyperkalemia necessitating the interruption of ACEi/ARB therapy developed in $19 \%$ of patients. This suggests great caution in the widest utilization of this class of drugs in HD patients.

(c) 2018 S. Karger AG, Basel

\section{Introduction}

Cardiovascular disease is the primary cause of mortality and morbidity in the end-stage renal disease (ESRD) population and occurs in as many as $50-60 \%$ of ESRD patients [1]. Hypertension has been recognized as one of the principal contributors to death in these patients, its prevalence ranging from more than $80 \%$ in the United States [2], about 50\% in Great Britain [3], and 70\% in Italy [4]. The renin-angiotensin-aldosterone system (RAAS) plays key roles in the regulation of blood pressure, blood volume and cardiovascular function. Therapeutic manipulation of the RAAS by the use of angiotensin converting enzyme inhibitors (ACEi) and/or angiotensin receptor blockers (ARB) is an important treatment strategy for hypertension, heart failure, and diabetic patients.

In recent years, $\mathrm{ACEi}$ and $\mathrm{ARB}$ have been increasingly used in patients with ESRD with the aim to reduce fatal and nonfatal cardiovascular events and left ventricular mass [5]. However, recent evidence suggests that ACEi and ARB may not be superior to other antihypertensive agents in reducing cardiovascular risk in advanced kidney disease [6] and are often associated with an increased risk of hyperkalemia, particularly when administered in combination [7]. ACEi and ARB may have different effects on potassium levels that reflect differences in their actions on potassium homeostasis. Patients with diabetes, heart failure, or chronic renal failure treated with these drugs are at an increased risk of hyperkalemia compared with those without these conditions [8]. However, their effect on serum potassium $(\mathrm{sK})$ concentrations in anuric patients on chronic HD treatment is still controversial.
Increased sK levels may induce characteristic electrocardiographic changes that can vary depending on the severity and rapidity of sK changes. $\mathrm{sK} \geq 6 \mathrm{mmol} / \mathrm{L}$ are generally considered to be clinically significant and are estimated to complicate $1.4 \%$ of hospital admissions in general population $[9,10]$ and are associated with increased all-cause mortality, cardiovascular mortality, and arrhythmogenic death in HD patients [11]. The aim of the study has been to evaluate sK concentrations before and after ACEi/ARB therapy introduction in a cohort of prevalent anuric uremic patients on thrice weekly HD treatment.

\section{Patients and Methods}

A retrospective analysis of prospectively acquired data to evaluate $s \mathrm{~K}$ changes before and after the assumption of ACEi/ARB therapy was done from 1 January 2015 to 31 December 2015. The study was part of standard antihypertensive treatment in prevalent HD patients of the O.U. of Nephrology, ASST Spedali Civili and University of Brescia. The primary outcome was the evaluation of pre-HD sK and peak-sK concentration changes in the 3 months before and after the introduction of the ACEi/ARB therapy. The secondary outcome was the proportion of patients who developed moderate or severe kyperkalemia after ACEi/ARB therapy introduction, and the proportion of patients requiring the reduction in dialysate $\mathrm{K}$ concentration or drug discontinuation. The study protocol, conducted according to the principles of Declaration of Helsinki, has been approved by the institute's committee on human research. Informed consent was obtained from all study participants.

\section{Selection Criteria}

All the prevalent oligo-anuric patients on regular 3 times weekly HD treatment for at least 6 months who introduced ACEi or $\mathrm{ARB}$ as part of their current treatment of hypertension according to the attending doctor clinical judgment and prescription, double RAAS blockade (ACEi + ARB) therapy association was not allowed.

\section{Exclusion Criteria}

Those who had a stroke or myocardial infarction within the previous 3 months, active malignancy, or cachexia were not included in the study.

\section{Source of Data}

Data were acquired from a computerized data file that included all demographic, clinical, and laboratory data of the patients prospectively collected according to specific standard of care procedures followed in our Dialysis Unit.

\section{Study Protocol}

In the 3 months before ( $36 \mathrm{HD}$ sessions) and after ( $36 \mathrm{HD}$ sessions) the introduction of ACEi/ARB therapy, the following variables were evaluated in pre-dialysis after the long interdialysis interval: $\mathrm{m}$-sK (mean value of 12 determinations; mmol/L), peak-sK
Movilli/Camerini/Gaggia/Zubani/ Cancarini 
(maximum $\mathrm{K}$ value observed during observations the follow-up; $\mathrm{mmol} / \mathrm{L})$, serum sodium $(\mathrm{sNa} ; \mathrm{mmol} / \mathrm{L})$, pre dialysis systolic blood pressure (SBP; $\mathrm{mm} \mathrm{Hg}$ ) and diastolic blood pressure (DBP; mm $\mathrm{Hg}$ ), body weight (BW; Kg), interdialytic weight gain (IDWG; $\mathrm{Kg}$ ), serum bicarbonate concentrations $(\mathrm{mmol} / \mathrm{L})$. Kt/V and protein catabolic rate (PCRn; g/Kg BW/day) were assessed midweek at the start and at the end of observation. Kt/V was calculated according to Daugirdas [12], PCRn according to the K/DOQI clinical practice guidelines for nutrition in chronic renal failure [13]. Parameters like the type of dialysis, duration, blood and dialysate flow rate were kept constant during observation.

sK and sNa were measured by indirect ionometry using a Siemens v-Lyite ${ }^{\circledR}$ integrated multisensor system or a Dimension Vis$\mathrm{ta}^{\circledR}$ system. The other parameters were measured by standard laboratory methods. All patients were advised to eat a normal protein (1.0-1.2 g/kgBW/day), low salt (mean salt intake $4-5 \mathrm{~g} /$ day) diet. Meals or drinks during dialysis were not allowed.

Definitions Used in the Study

Residual Urine Output: Residual urine output was evaluated by 24-h urine collection during the long interdialytic interval at the time of enrolment. Oligo-Anuria was defined by residual renal output $<250 \mathrm{~mL} / 24 \mathrm{~h}$.

Hyperkalemia [9]: Pre-HD sK $>6 \mathrm{mmol} / \mathrm{L}$ was considered severe hyperkalemia requiring the suspension of $\mathrm{ACEi} / \mathrm{ARB}$ therapy; $5.5 \geq \mathrm{sK} \leq 6 \mathrm{mmol} / \mathrm{L}$ was considered mild hyperkalemia treatable with a modification of $\mathrm{K}$ in the dialysate without ACEi/ARB therapy suspension.

Pre-HD SBP and DBP: These values were obtained by trained dialysis nurses, using an automated device in the upper arm opposite to fistula after the patient was at rest for $5 \mathrm{~min}$ in a supine or sitting position. The values used in the analysis were the mean of pre-HD SBP and DBP for each patient over the 3 months of observation (36 HD sessions).

Dry Body Weight: BW was clinically determined and reflects the lowest weight the patient can tolerate without intradialytic symptoms and hypotension in the absence of overt fluid overload.

Interdialytic Weight Gain: IDWG was the difference between pre-HD weight and post-HD weight of the previous HD session. The value of IDWG used in the analysis was the mean IDWG for each patient over the 3 months of observation (36 HD sessions).

Data Analysis

Continuous data are reported as mean \pm SD or median and interquartile range according to their distribution. Comparisons between patients were performed using Student $t$ test for paired and unpaired data for normally distributed data, Mann-Whitney test for medians, and $\chi^{2}$ test for categorical data. Significant differences were defined by $p<0.05$. All statistical analyses were performed using Statgraphics Centurion for Windows software (Statgraphics Centurion ${ }^{\circledR}$ ).

\section{Results}

From a total prevalent HD population of 240 patients, 112 patients ( 67 men, 45 women), mean age $67 \pm 14$ years (range 22-89), on regular chronic HD treatment for
Table 1. Baseline clinical and laboratory characteristics of the patients studied

Patients on study 112

Age, years, mean \pm SD

$67 \pm 14$

Male gender, $n(\%)$

Dialysis vintage, months*

$67(60)$

Cardiovascular disease, $n(\%)$

$44(6-212)$

Diabetes, $n$ (\%)

$45(40)$

$20(18)$

Antihypertensive therapy, $n(\%)$

$101(90)$

$69 \pm 17$

$50(0-200)$

Urinary output, $\mathrm{mL} / 24 \mathrm{~h}^{*}$

$2.9 \pm 1.0$

$132 \pm 8$

$72 \pm 10$

77 (69)

$35(31)$

$\mathrm{HD}, n(\%)$

HDF-ol, $n(\%)$

$\mathrm{Kt} / \mathrm{V}$, mean $\pm \mathrm{SD}$

$1.46 \pm 0.21$

PCRn, g/KgBW/day, mean \pm SD

$1.04 \pm 0.26$

* Median (IQR).

IDWG, interdialytic weight gain; Pre HD SBP, pre dialysis systolic blood pressure; Pre HD DBP, pre-dialysis diastolic blood pressure; HD, bicarbonate hemodialysis; HDF-ol, online hemodiafiltration; IQR, interquartile range.

6-212 months (median 44 months), met the inclusion/ exclusion criteria. Underlying renal diseases included chronic glomerulonephritis in 13 patients, renal vascular disease in 19 patients, diabetes in 20 patients, tubule-interstitial nephritis in 11 patients, polycystic kidney disease in 13 patients, and undiagnosed nephropathy in 36 patients.

Seventy-seven patients (pts) were on bicarbonate HD with high-flux synthetic membranes (Helixone FX80, Revaclear 400), 35 on online-HDF with high-flux synthetic membranes (Helixone FX Cordiax, Polyflux 210H). Blood flow rate ranged from 250 to $350 \mathrm{~mL} / \mathrm{min}$ (median 300); dialysate flow rate was $500 \mathrm{~mL} / \mathrm{min}$. Dialysate fluid composition was sodium $140 \mathrm{mmol} / \mathrm{L}$, potassium 2-3 mmol/L, calcium $1.5 \mathrm{mmol} / \mathrm{L}$, bicarbonate $32 \mathrm{mmol} / \mathrm{L}$, acetate $4 \mathrm{mmol} / \mathrm{L}$, and glucose $1 \mathrm{~g} / \mathrm{L}$.

Table 1 shows the baseline clinical and laboratory characteristics of the 112 patients treated with ACEi/ARB drugs. Median urinary output was $50 \mathrm{~mL} / 24 \mathrm{~h}$ (IQ range 0-200 mL/24 h). Cardiovascular disease was present in $40 \%$ of the patients and diabetes was in $18 \%$. One hundred 2 patients (91\%) were already on anti-hypertensive therapy. Of these patients, 26 pts (23\%) were treated with beta blocker, 41 pts (37\%) with calcium channel blocker, 15 pts (13\%) with calcium channel blocker + beta blocker, and 20 pts (18\%) with doxazosin or clonidine. 
Table 2. Type and dosage of renin-angiotensin-aldosterone system blocker drugs in the 112 patients under study

\begin{tabular}{llc}
\hline Patients on ACEi & 53 & \\
\hline Drug & patients, $n(\%)$ & dose, mg* \\
\hline Ramipril & $41(37)$ & $5(2.5-10)$ \\
Enalapril & $11(10)$ & $10(5-20)$ \\
\hline Patients on ARB & 59 & \\
\cline { 2 - 3 } & patients, $n(\%)$ & dose, mg* \\
\hline Losartan & $44(39)$ & $50(25-100)$ \\
Valsartan & $16(14)$ & $80(40-80)$ \\
\hline
\end{tabular}

* Median (IQR). IQR, interquartile range.

Table 2 shows the type and dosage of ACEi/ARB therapy: 53 out of $112(47 \%)$ of the patients were treated with an ACEi and 59 out of $112(53 \%)$ were treated with an ARB. No change in dosage was done. None had either drug in combination.

\section{Change in sK Concentrations}

Table 3 shows the pre-HD m-sK and peak-sK before and after 3 months of therapy in patients treated with ACEi or ARB drugs. Both $\mathrm{m}$-sK and peak-sKmax increased significantly in both groups of patients. There was no significant difference in pre-HD $\mathrm{m}$-sK concentrations between patients treated with $\mathrm{ACEi}$ or $\mathrm{ARB}$ drugs either before or after the introduction of each class of drug. For this reason, we considered all patients as a whole group in further analysis.

Figure 1a shows the m-sK pre-HD of the whole group of patients before ACEi/ARB introduction and after 3 months of ACEi/ARB therapy; $\mathrm{m}$-sK increased from 5.0 \pm 0.4 to $5.7 \pm 0.6 \mathrm{mmol} / \mathrm{L}(p<0.0001)$. Peak-sK increased from $5.3 \pm 0.5$ to $6.2 \pm 0.6 \mathrm{mmol} / \mathrm{L}$ ( $p<0.0001$; Fig. $1 \mathrm{~b})$.

Figure 2 shows the proportion of patients with normal $\mathrm{m}$-sK and mild or severe hyperkalemia before and after ACEi/ARB therapy. Patients with normal serum K concentrations decreased from 92 out of $112(82 \%)$ before ACEi/ARB therapy to 33 out of 112 (29\%) after ACEi/ARB therapy $(p<0.0001)$. Mild hyperkalemia increased from 20 out of 112 patients (18\%) to 59 out of $112(52 \% ; p<0.001)$. None of the patients had severe hyperkalemia before ACEi/ARB therapy, but after 3 months of ACEi/ARB therapy, 22 out of 112 (19\%) developed severe hyperkalemia $(p<0.0001)$ necessitating drug interruption.
Pre-HD m-sK concentrations in patients that developed mild hyperkalemia varied from $5.1 \pm 0.3 \mathrm{mmol} / \mathrm{L}$ before ACEi/ARB therapy to $5.8 \pm 0.2(p<0.0001)$ after 3 months of ACEi/ARB therapy. None of these patients withdrew ACEi/ARB therapy, but 20 out of 63 (31\%) reduced dialysate $\mathrm{K}$ concentration (from 3 to $2 \mathrm{mmol} / \mathrm{L}$ ).

Figure 3 shows the pre-HD m-sK and peak-sK in the 22 patients that developed severe hyperkalemia before and at the time of ACEi/ARB therapy suspension (on average $1.5 \pm 0.4$ months after the start of therapy). $\mathrm{m}$-sK varied from $5.2 \pm 0.3 \mathrm{mmol} / \mathrm{L}$ before ACEi/ARB therapy to $6.5 \pm 0.2 \mathrm{mmol} / \mathrm{L}$ at the moment of suspension of therapy $(p<0.0001)$. Peak-sK varied from $5.5 \pm 0.3 \mathrm{mmol} / \mathrm{L}$ before ACEi/ARB introduction to $6.9 \pm 0.3 \mathrm{mmol} / \mathrm{L}$ at the moment of suspension of therapy $(p<0.0001)$. After the suspension of ACEi/ARB therapy, m-sK returned to basal levels within 1 month (from $6.5 \pm 0.2$ to $5.2 \pm$ $0.4 \mathrm{mmol} / \mathrm{L} ; p<0.0001$ ).

\section{Blood Pressure, Serum Sodium, Body Weight,} Interdialytic Weight Gain, Serum Bicarbonate, $K t / V$, and Normalized Protein Catabolic Rate Changes

Table 4 shows the values of pre-HD SBP and DBP, serum $\mathrm{Na}$ concentrations, BW, IDWG, Kt/V, serum bicarbonate concentrations, and nPCR in the 112 patients treated with ACEi or ARB drugs. No significant changes were observed.

\section{Discussion}

Our study clearly indicates that in prevalent anuric uremic patients on regular thrice weekly hemodialysis, ACEi or ARB therapy may result in a significant increase in pre-HD $\mathrm{m}$-sK that lead to severe hyperkalemia, necessitating therapy suspension, in $19 \%$ of cases. Both ACEi and $\mathrm{ARB}$ are considered effective medications that can decrease cardiovascular morbidity and mortality with mechanisms partially independent from the normalization of high blood pressure $[14,15]$. As such, their clinical use is very common even in HD patients. However, data concerning the side effect of hyperkalemia in patients on regular HD are still controversial [16]. For this reason, we conducted this observational study with the aim of evaluating the pre HD serum $\mathrm{K}$ concentrations after the introduction of an ACEi or an ARB as part of standard antihypertensive therapy in prevalent anuric HD patients. The vast majority of our patients (91\%) were already on an antihypertensive treatment, most commonly with a calcium channel blocker and/or a beta
Movilli/Camerini/Gaggia/Zubani/ Cancarini 
Fig. 1. Pre-dialysis mean serum potassium concentrations (a) and maximum serum potassium concentrations $(\mathbf{b})$ of the whole group of patients before ACEi/ARB introduction and after 3 months of ACEi/ARB therapy.

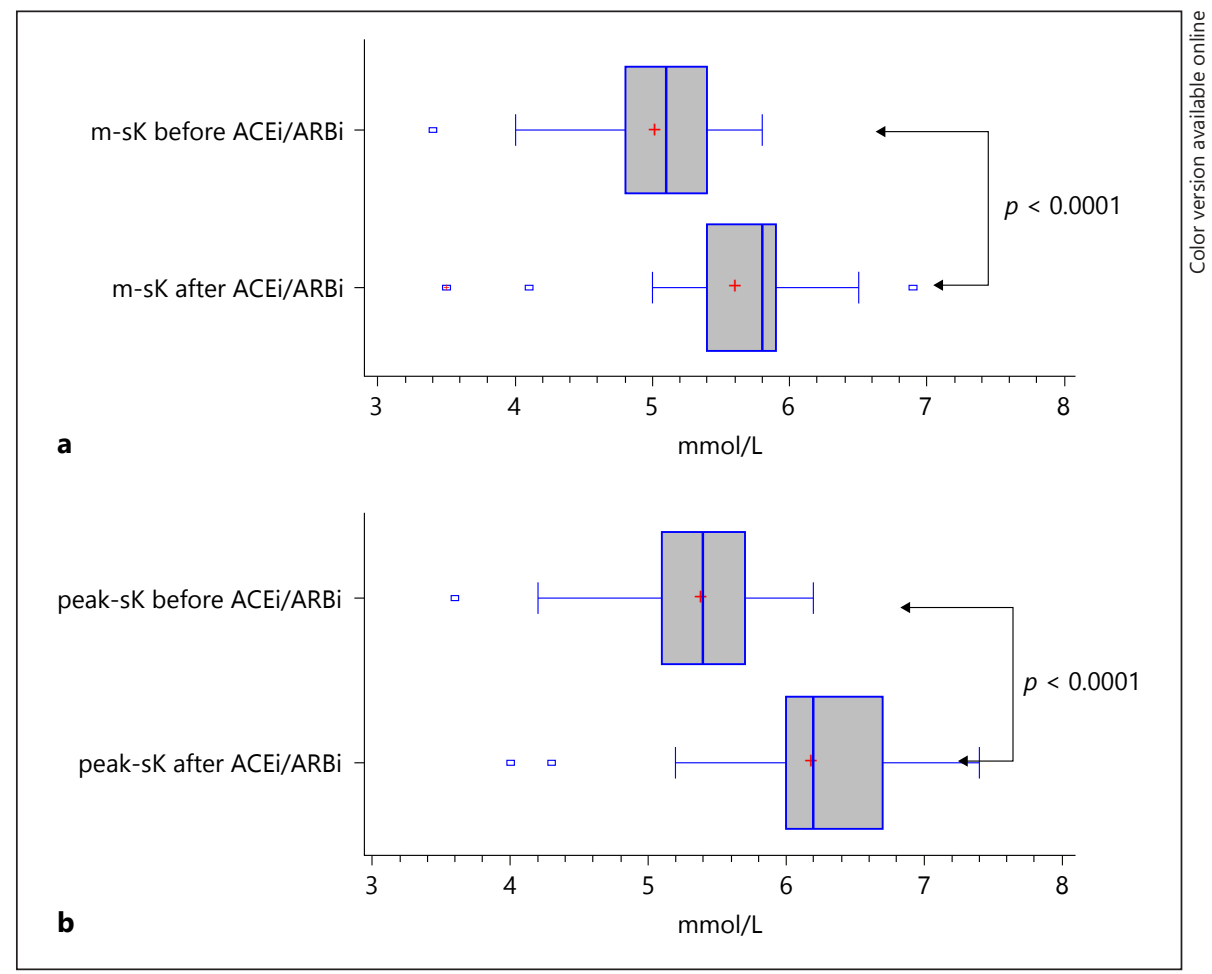

Table 3. Pre-HD plasma potassium and plasma potassium max concentrations before and after 3 months of therapy in the 112 patients treated with ACEi or ARB drugs

\begin{tabular}{|c|c|c|c|c|c|c|c|}
\hline & & $\begin{array}{l}\mathrm{m} \text {-sK before } \\
\mathrm{mmol} / \mathrm{L}\end{array}$ & $\begin{array}{l}\mathrm{m} \text {-sK after } \\
\mathrm{mmol} / \mathrm{L}\end{array}$ & $p$ value & $\begin{array}{l}\text { Peak-sK before } \\
\mathrm{mmol} / \mathrm{L}\end{array}$ & $\begin{array}{l}\text { Peak-sK after } \\
\mathrm{mmol} / \mathrm{L}\end{array}$ & $p$ value \\
\hline Patients on ACEi & 53 & $5.1 \pm 0.3$ & $5.7 \pm 0.5$ & $<0.0001$ & $5.4 \pm 0.3$ & $6.3 \pm 0.3$ & $<0.0001$ \\
\hline Patients on ARB & 59 & $5.0 \pm 0.5$ & $5.6 \pm 0.6$ & $<0.0001$ & $5.3 \pm 0.6$ & $6.2 \pm 0.4$ & $<0.0001$ \\
\hline$p$ (ACEi vs ARB) & & NS & NS & & NS & NS & \\
\hline
\end{tabular}

NS, not significant.

blocker. We decided to introduce an ACEi/ARB drug at low dosage (Table 2) because randomized [14] and clinical [17] trials suggested that the inhibition of the RAAS could contribute to reduce morbidity and mortality in dialyzed uremic patients and could improve left ventricular hypertrophy even independently from their hypotensive effect. As such, as shown in Table 4, in our patients, systolic and diastolic blood pressure levels did not change significantly, thereby showing on the average a good blood pressure control either before or after the introduction of ACEi/ARB treatment [18]. Notwithstanding this (Fig. 1a, b), 3 months of ACEi or ARB assumption resulted in a significant increase in pre-HD $\mathrm{m}$-sK independent on the type of RAAS blocker used. In fact, as shown in Table 3 , the magnitude of the increment in serum $\mathrm{K}$ concentrations, was very similar in patients treated with an ACEi or an ARB drug, and this was further confirmed by the fact that serum $\mathrm{K}$ concentrations either before or after the introduction of each class of drugs were not significantly different in the 2 groups of patients. Moreover, as shown in Figure 2, the proportion of patients with normal serum $\mathrm{K}$ concentrations decreased sharply from $82 \%$ before ACEi/ARB therapy to $29 \%$ after ACEi/ARB therapy $(p<0.0001)$, while the proportion of those with mild hyperkalemia $(5.5 \geq \mathrm{sK}$ $\leq 6.0 \mathrm{mmol} / \mathrm{L}$ ), increased from $18 \%$ before to $52 \%$ after ACEi/ARB therapy $(p<0.001)$. According to our protocol, ACEi/ARB were not stopped, but in 20 out of 63 patients $(31 \%)$, it was necessary to reduce the dialysate $\mathrm{K}$ concentration from 3 to $2 \mathrm{mmol} / \mathrm{L}$. 


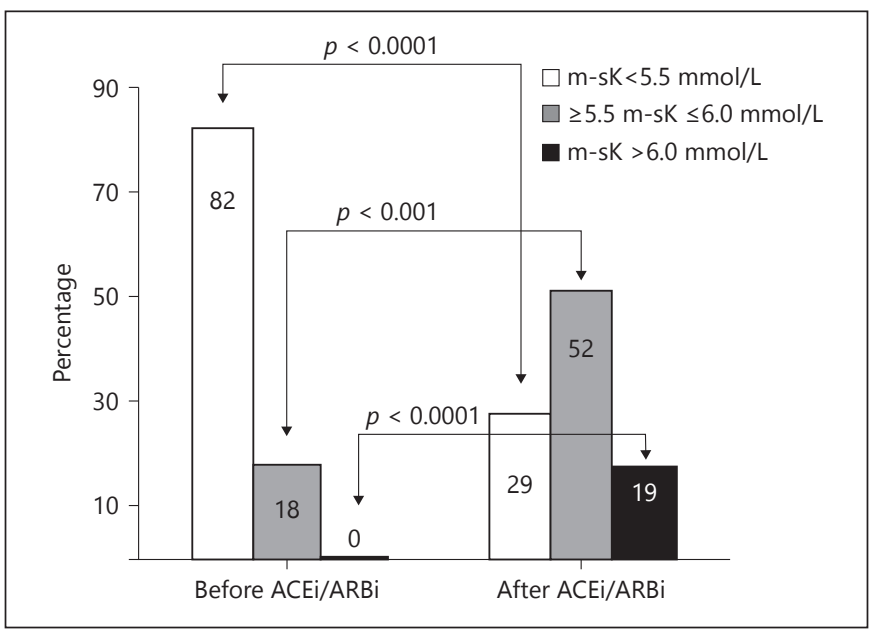

Fig. 2. Proportion of patients with normal serum potassium concentrations, mild and severe hyperkalemia before and after ACEi/ ARB therapy.

Another important observation was the finding that while none of the patients had severe hyperkalemia (sK $>6 \mathrm{mmol} / \mathrm{L}$ ) before ACEi/ARB therapy, $19 \%$ of the patients developed this complication after ACEi/ARB introduction $(p<0.0001)$ necessitating treatment interruption. In these patients (Fig. 3), m-sK and peak-sK varied from $5.2 \pm$ 0.3 and $5.5 \pm 0.3 \mathrm{mmol} / \mathrm{L}$ before ACEi/ARB therapy to $6.5 \pm 0.2$ and $6.9 \pm 0.3 \mathrm{mmol} / \mathrm{L}$ at the moment of suspension of therapy $(p<0.0001$; on the average 1.5 months from the start of treatment). This sequence of events is in agreement with reports of hyperkalemia following the blockade of the RAAS in patients with chronic renal failure still not on dialysis [19] and confirm previous data from Knoll et al. [20] that the risk of hyperkalemia continues even after regular dialysis therapy has been initiated and diuresis is lost. Since our patients had virtually no residual renal function, the observed increased risk of hyperkalemia is consistent with previous studies suggesting that the blockade of RAAS may have important effects on the extra-renal handling of potassium homeostasis. In a study involving anephric dialysis patients, the disposal of an acute potassium load was enhanced by mineralocorticoid administration and impaired by spironolactone suggesting a role of aldosterone on extrarenal cellular uptake of potassium [21]. Other studies have shown that intestinal potassium excretion is increased significantly in patients with renal failure [22], supporting the hypothesis that patients with endstage renal disease adapt to decreased renal potassium excretion by increasing colonic potassium secretion. However, despite these adaptations, oligo anuric patients have

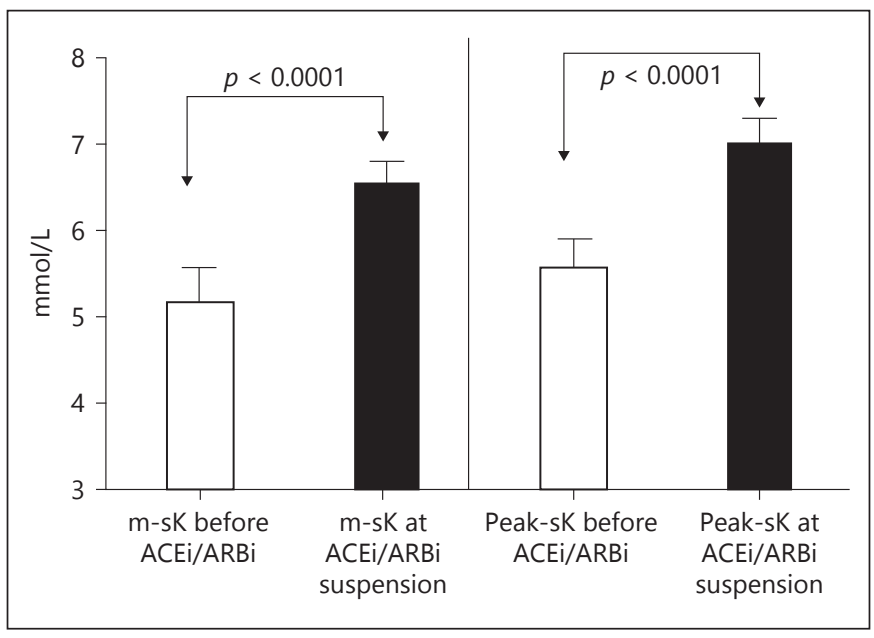

Fig. 3. Pre-dialysis mean serum potassium and maximum serum potassium concentrations in patients who developed severe hyperkalemia before and at the time of ACEi/ARB therapy suspension.

limited reserve to tolerate any additional insult [23]. Addition of ACEi/ARB therapy can increase the risk of hyperkalemia interfering with the colonic secretion [24] and the extracellular removal of potassium [21]. This seems to be the most likely explanation of the phenomenon given that, as shown in Table 4, there were no concomitant significant variations in body weight, interdialytic weight gain, $\mathrm{Kt} / \mathrm{V}$, serum $\mathrm{Na}$ concentrations, serum bicarbonate concentrations, and protein catabolic rate either before or after ACEi/ARB treatment, thus excluding that each one of these variables could have exerted any significant influence on the variation in sK concentrations. In further support to this assumption is the observation that in these patients, the suspension of ACEi/ARB therapy returned sK concentrations to basal levels within 1 month (sK from $6.5 \pm 0.2$ to $5.2 \pm 0.4 \mathrm{mmol} / \mathrm{L} ; p<0.0001)$. We chose to interrupt ACEi/ARB administration in patients who developed severe hyperkalemia due to the lack of effective agents for outpatient treatment of this condition [25]. Sodium polystyrene sulfonate may cause serious gastrointestinal adverse events that limit its extended use [26]. It is possible that the introduction of more effective and well-tolerated oral agents could change the future approach [23].

Our study has some strengths including the fact that patients were controls of their own, thus minimizing the possible confounding originating from individual differences in dietary habits or seasonal variations in potassium consumption, and other unmeasured variables. However, the study also has some limitations including the fact that patients were not randomized. As a result, it could be that 
Table 4. Pre-HD systolic and diastolic blood pressure, serum sodium concentrations, body weight, interdialytic weight gain, $\mathrm{Kt} / \mathrm{V}$, serum bicarbonate concentrations, and normalized protein catabolic rate before and after 3 months of ACEi/ARB treatment in the 112 patients treated with ACEi or ARB drugs

\begin{tabular}{lccc}
\hline & Before ACEi/ARB & After ACEi/ARB & $p$ value \\
\hline PAS, mm Hg & $133 \pm 8$ & $132 \pm 9$ & NS \\
PAD, mm Hg & $72 \pm 10$ & $71 \pm 9$ & NS \\
Serum Na, mmol/L & $139.0 \pm 1.3$ & $138.3 \pm 1.3$ & NS \\
Body weight, Kg & $68 \pm 17$ & $68 \pm 17$ & NS \\
IDWG, Kg & $2.9 \pm 1.0$ & $3.1 \pm 1.0$ & NS \\
Kt/V & $1.46 \pm 0.21$ & $1.45 \pm 0.20$ & NS \\
Serum bicarbonate, mmol/L & $23.2 \pm 1.3$ & $23.4 \pm 1.4$ & NS \\
PCRn, g/KgBW/day & $1.04 \pm 0.26$ & $1.05 \pm 0.25$ & NS
\end{tabular}

NS, not significant.

some patients with recurrent hyperkalemia at the time the study was started may have been excluded, thus introducing a possible selection bias. However, such a confounding would tend to weaken rather than to potentiate the observed association between RAAS blockade and hyperkalemia, and this gives further strength to our observations.

\section{Conclusion}

Treatment with ACEi/ARB is associated with an increased risk of hyperkalemia in anuric hemodialysis patients. The proportion of patients with normal m-sK con- centrations decreased from 82 to $29 \%$ and those with mild hyperkalemia increased from 18 to $52 \%$, necessitating in $31 \%$ of cases the reduction of $\mathrm{K}$ concentration in the dialysate. Severe hyperkalemia necessitating interruption of ACEi/ARB therapy developed in 19\% of patients. This suggests great caution in the increasingly wider utilization of this class of drugs in anuric HD patients.

\section{Disclosure Statement}

Authors declare that they have no sponsorship or funding arrangements relating to their research and no possible conflicts of interest to disclose.

\section{References}

1 Go AS, Chertow GM, Fan D, McCulloch CE, Hsu C: Chronic kidney disease and the risks of death, cardiovascular events, and hospitalization. N Engl J Med 2004;351:1296-1305.

2 Agarwal R, Nissenson AR, Batlle D, et al: Prevalence, treatment, and control of hypertension in chronic hemodialysis patients in the United States. Am J Med 2003;115:291297.

3 Rao A, Pitcher D, Farrington K: UK Renal Registry 16th annual report: chapter 11 blood pressure profile of prevalent patients receiving renal replacement therapy in 2012: national and centre-specific analyses. Nephron Clin Pract 2013;125:209-218.

4 Del Vecchio L, Lusenti T, Del RG et al: Prevalence of hypertension in a large cohort of Italian hemodialysis patients: results of a cross-sectional study. J Nephrol 2013:26: 745-754.

5 Tai DJ, Lim TW, James MT, et al: Cardiovascular effects of angiotensin converting en- zyme inhibition or angiotensin receptor blockade in hemodialysis: a meta-analysis. Clin J Am Soc Nephrol 2010;5:623-630.

6 Ahmed A, Jorna T, Bhandari S: Should we STOP angiotensin converting enzyme inhibitors/angiotensin receptor blockers in advanced kidney disease? Nephron 2016;133:147-158.

7 Takaichi K, Takemoto F, Ubara Y, Mori Y: Analysis of factors causing hyperkalemia. Intern Med 2007;46:823-829.

8 Acker CG, Johnson JP, Palevsky PM, Greenberg A: Hyperkalemia in hospitalized patients: causes, adequacy of treatment, and results of an attempt to improve physician compliance with published therapy guidelines. Arch Intern Med 1998;158:917-924.

9 Paice B, Gray JM, Mc Bride D, Donnelly T, Lawson $\mathrm{DH}$ : Hyperkalaemia in patients in hospital. Br Med J (Clin Res Ed) 1983;286:1189-1192.

10 Kovesdy CP: Management of hyperkalaemia in chronic kidney disease. Nat Rev Nephrol 2014;10:653-662.
11 Sanghavi S, Whiting S, Uribarri J: Potassium balance in dialysis patients. Semin Dial 2013; 26:597-603.

12 Daugirdas JT: Second generation logarithmic estimates of single-pool variable volume $\mathrm{Kt} / \mathrm{V}$ : an analysis of error. J Am Soc Nephrol 1993;4: 1205-1213.

13 NKF-DOQI Nutrition Work Group: Clinical Practice Guidelines for Nutrition. In Chronic Renal Failure. New York, National Kidney Foundation 2001, pp 75-80.

14 Savarese G, Costanzo P, Cleland JC, et al: A meta-analysis reporting effects of angiotensin converting enzyme inhibitors and angiotensin receptor blockers in patients without heart failure. J Am Coll Cardiol 2013;61:131-142.

15 Shih CJ, Chu H, Ou SM, Chen YT: Comparative effectiveness of angiotensin-converting enzyme inhibitors and angiotensin II receptor blockers on major adverse cardiac events in patients with newly diagnosed type 2 diabetes: a nationwide study. Int J Cardiol 2015;199:283-289. 
16 Karaboyas A, Zee J, Brunelli SM, et al: Dialysate potassium, serum potassium, mortality, and arrhythmia events in hemodialysis: results from the Dialysis Outcomes and Practice Patterns Study (DOPPS). Am J Kidney Dis 2017;69:266-277.

17 Cannella G, Paoletti E, Delfino R, et al: Prolonged therapy with ACE inhibitors induces a regression of left ventricular hypertrophy of dialyzed uremic patients independently from hypotensive effects. Am J Kidney Dis 1997;30: 659-664.

18 Agarwal R: Blood pressure and mortality among hemodialysis patients. Hypertension 2010;55:762-76.

19 Weir MR, Rolfe M: Potassium homeostasis and renin-angiotensin-aldosterone system inhibitors. Clin J Am Soc Nephrol 2010; 5:531-548.
20 Knoll GA, Sahgal A, Nair RC, Graham J, van Walraven C, Burns KD: Renin-angiotensin system blockade and the risk of hyperkalemia in chronic hemodialysis patients. Am J Med 2002;112:110-114.

21 Sugarman A, Brown RS: The role of aldosterone in potassium tolerance: studies in anephric humans. Kidney Int 1988;34:397-403.

22 Sandle GI, Gaiger E, Tapster S, Goodship TH: Evidence for large intestinal control of potassium homoeostasis in uraemic patients undergoing long-term dialysis. Clin Sci (Lond) 1987;73:247-252.
23 Kovesdy CP, Appel LJ, Grams ME, et al: Potassium homeostasis in health and disease: A scientific workshop cosponsored by the National Kidney Foundation and the American Society of Hypertension. Am J Kidney Dis 2017;70:844-858.

24 Hatch M, Freel RW, Vaziri ND: Local upregulation of colonic angiotensin II receptors enhances potassium excretion in chronic renal failure. Am J Physiol 1998;274:F275F282.

25 Palmer BF: Managing hyperkalemia caused by inhibitors of the renin-angiotensin-aldosterone system. N Engl J Med 2004;351:585592.

26 Harel Z, Harel S, Shah PS, et al: Gastrointestinal adverse events with sodium polystyrene sulfonate (Kayexalate) use: a systematic review. Am J Med 2013;126:264.e9-e24. 